\title{
Strategic decision making under climate change: a case study on Lake Maggiore water system
}

\author{
M. MICOTTI ${ }^{1}$, R. SONCINI SESSA ${ }^{1} \&$ E. WEBER ${ }^{2}$ \\ 1 Dept. Electronics, Information and Bioengineering, Politecnico di Milano, Italy \\ micotti@elet.polimi.it \\ 2 Fondazione Politecnico di Milano, Italy
}

\begin{abstract}
Water resources planning processes involve different kinds of decisions that are generally evaluated under a stationary climate scenario assumption. In general, the possible combinations of interventions are mutually compared as single alternatives. However, the ongoing climate change requires us to reconsider this approach. Indeed, what have to be compared are not individual alternatives, but families of alternatives, characterized by the same structural decisions, i.e. by actions that have long-term effects and entail irrevocable changes in the system. The rationale is that the structural actions, once they have been implemented, cannot be easily modified, while the management decisions can be adapted to the evolving conditions. This paper considers this methodological problem in a real case study, in which a strategic decision has to be taken: a new barrage was proposed to regulate Lake Maggiore outflow, but, alternatively, either the present barrage can be maintained with its present regulation norms or with a new one. The problem was dealt with by multi-criteria decision analysis involving many stakeholders and two decision-makers. An exhaustive set of indicators was defined in the participatory process, conducted under the integrated water resource management paradigm, and many efficient (in Pareto sense) regulation policies were identified. The paper explores different formulations of a global index to evaluate and compare the effectiveness of the classes of alternatives under both stationary and changing hydrological scenarios in order to assess their adaptability to the ongoing climate change.
\end{abstract}

Key words decision making; climate change; multi-criteria analysis; reservoir planning and management

\section{INTRODUCTION}

The many different actions considered in water resource planning differ in timing, costs, impacts and complexity. Usually these actions are evaluated under a stationary climate scenario in which all the possible combinations of actions are mutually compared as single alternatives. However, the ongoing climate change requires us to re-consider this paradigm since structural decisions have longterm effects and entail irrevocable changes in the system, while management decisions can be progressively adapted to evolving climate and social conditions. Therefore in strategic decision making it appears more useful to not compare individual alternatives, but classes of alternatives that are characterized by the same mix of structural actions.

The classical Multi Attribute Value Theory (MAVT, Keeney and Raiffa 1976) is well suited for comparing individual alternatives, but it is not equipped to deal with strategic decisions in the sense mentioned above, i.e. for comparing classes of alternatives. In the authors' knowledge, the literature has not yet offered any solution solving it.

In this paper we propose to tackle the problem still remaining in the conceptual frame of MAVT, by associating each alternative with a suitable global value and then to compare the classes on the basis of the population of those values.

In order to introduce the problem we present in the next section a planning study on Lake Maggiore in a climate change prospective. In the third section a method for comparing classes of alternatives is presented and its significance is evaluated in the fourth one by applying it to the Lake Maggiore case. Concluding remarks close the paper.

\section{THE LAKE MAGGIORE CASE-STUDY}

Lake Maggiore (locally named Verbano) is a cross-border lake, shared between Italy and Switzerland, set among the spectacular peaks of the Central Alps. We consider its water system as composed of the lake itself, its catchment and the River Ticino that flows out of the lake. The lake is regulated by a barrage, the Miorina barrage, which is managed on the basis of an international agreement, signed between Italy and Switzerland in 1940. The agreement states that the outflow from the lake may be regulated only when the lake level (measured at Sesto Calende hydrometer) is within a suitable, time- 
varying regulation range $([-0.5 \mathrm{~m}, 1 \mathrm{~m}]$ on Sesto hydrometer from March to October, and $[-0.5 \mathrm{~m}$, $1.5 \mathrm{~m}]$ in the rest of the year). Outside of this range the lake outflow must be set to specified values. Namely, when the lake level equals the minimum of the regulation range, the outflow must be set equal to the lake inflow, in order to prevent further dropping of the level; while, when the level equals or exceeds the maximum of the regulation range, the outflow must be the largest feasible one, i.e. the barrage gates should be completely open, in order to minimize the flood peak on the lake.

The planning study we dealt with concerns the evaluation of the opportunity of substituting the Miorina barrage with a new one and/or the identification of a new regulation policy. More precisely we consider the following classes of alternatives:

Class A: The Miorina barrage remains unchanged, as well as the regulation range; the daily release is defined by a regulation policy, when the lake level is within the regulation range; when it is outside of it the present constraints remain valid.

Class B: As class A, but for the regulation range that is assumed time invariant and equal to $[-0.5 \mathrm{~m}$, $1.5 \mathrm{~m}]$.

Class C: The Miorina barrage is substituted by the new barrage proposed by AIPO (Agenzia Interregionale fiume PO) and a stretch of the River Ticino is dredged (Da Deppo and Salandin 2006); the regulation range has no upper limit and thus the new regulation policy has to be defined for all possible lake levels. As can be seen in Fig. 1 these structural interventions will significantly enlarge the regulation possibilities and, consequently, the relevance of the regulation policy.

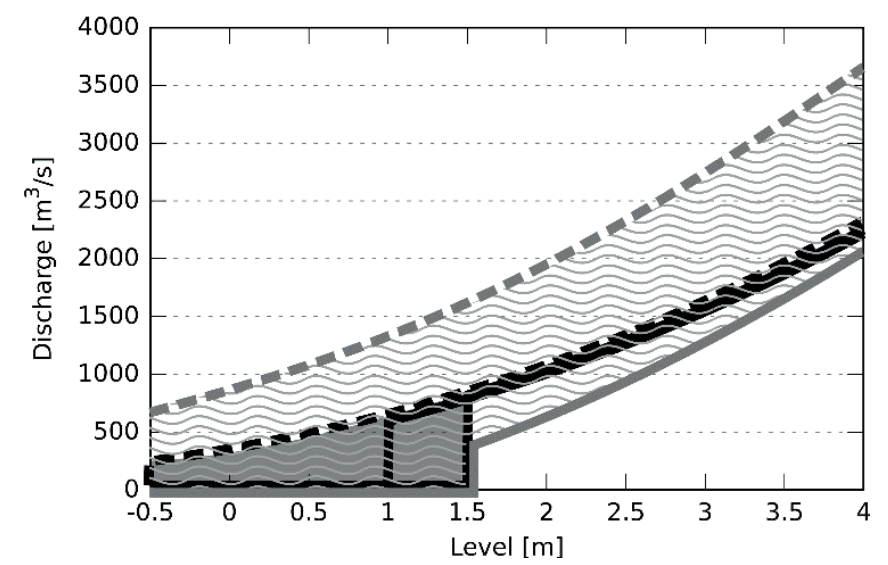

Fig. 1 Stage-discharge relationships for Lake Maggiore: black lines are related to the present barrage, the grey ones to the new barrage; the continuous lines concern the minimum release, the dashed ones the maximum. The filled area is the present regulation space, the wavy area the future one.

A model of each component of the system was identified and implemented in the TwoLe suite (Soncini-Sessa et al. 2003), a DSS to design regulation policies using the Dynamic Programming algorithm (Bellman 1957). More than 1300 Pareto-efficient alternatives were identified within the three classes, focusing the investigation in the more promising compromise area.

\section{The participatory approach}

The identification of the criteria hierarchy considered in the planning study was carried out together with the stakeholders, who were involved in the negotiations later on, following an integrated approach to participation (Reed 2008). The hierarchy reflects the complexity of the system: a set of 120 indicators has been identified and clustered into 20 different sectors, representing interests and stakeholders playing in the system (e.g. the indicator Maximum flooded area over the time horizon for Lake Flood sector or the indicator Average distance from natural hydrologic regime for River Environment sector). The effects induced by each one of 1300 Pareto efficient alternatives were therefore measured by a vector of 20 values, one for each sector, computed with suitable value functions on the basis of the indicators' outcomes. 
The evaluation of the indicators was based on a stationary hydrological scenario, more specifically on the time series of the historically registered inflows, in order to give stakeholders the possibility of comparing the performances of the alternatives with respect to the effects of the historical regulation, of which they have a memory.

Since the classes of alternatives include the possibility of a structural modification, which will have long-term effects, the evaluation on the stationary scenario is insufficient due to possible climate change. Hydrologic scenarios under climate change were therefore generated (the generation is due to R. Bordoy and P. Burlando, Zurich ETH), involving downscaling time series produced by the regional model REG-CM3 (Pal et al.; 2007), fed by global scenarios generated by model A1b (IPCC 2007). Ten equiprobable rainfall and temperature time series for the control period and ten for the decade 2041-2050 were thus obtained. These scenarios were then routed through the spatially distributed rainfall-runoff model FEST-WB (Ravazzani et al. 2008), to obtain the corresponding hydrologic scenarios, which were used to simulate the performances of the system under the 1300 alternatives. The indicators so obtained were used to assess the ratio of variation between the sector values evaluated on the decades 2041-2050 and the ones evaluated on the corresponding control scenario. These ratios were finally used to project the sector values estimated with the present stationary scenario to the decade 2041-2050; in so doing the bias that is present in the climate models, and therefore in the sector values evaluated with their outputs, was removed.

\section{A METHOD FOR COMPARING CLASSES OF ALTERNATIVES}

The information produced with a planning process based on classical MAVT can feed negotiation rounds, at the end of which, one (or a restricted number of) appealing compromise alternative(s) is (are) identified. When the future scenario is perceived as stationary, this choice is well based. In contrast, in the presence of an ongoing climate change the goal is not to identify the best compromise alternatives among the many efficient ones, but to select one class of alternatives, since, as mentioned in the introduction, the structural actions will affect the system in the long run, but the management policy can be subject to revision. Therefore the choice of one class cannot rely on a specific regulation policy, i.e. on one specific alternative, but it must consider all of them in some suitable sense.

Our proposal is to compare classes of actions with a procedure in two steps: by first defining a global value function in order to associate a global value to each alternative (as in MAVT) and then comparing the classes on the basis of the population of the global values they produce.

\section{Formulation of the global value function}

The idea is classical: to associate a global value $V_{j . A}$ to the $j$ th alternative of class $A$ by aggregating its sector values $V_{j . A}^{S}$ for all the sectors $s$. MAVT suggests consideration of their weighted sum, but a well-defined way of setting these weights does not exist when a compromise alternative does not have to be selected, as in our case.

The formulations we proposed are based on the assumption that no compensation is possible either among the sectors (Formulation 1) or among groups of sectors (Formulation 2). In that case it was proved (Keeney and Raiffa 1976, Triantaphyllou and Baig 2005) that a suitable form of the global value function is the (eventually weighted) product of the sector values. Then, assuming all weights equal to 1 , our first proposal is the following:

\section{Formulation 1:}

$$
V_{j, A}^{1}=\prod_{s \in S} \frac{v_{j, A}^{s}}{v_{0}^{s}}
$$

where $v_{\mathrm{o}}^{s}$ is the $s$ th sector value for the reference alternative $A 0$ and $S$ is the sector set.

Formulation 1 implies that when an alternative, say the $j$ th alternative of class $A$, completely dissatisfies sector $s$, i.e. when $v_{j, A}^{s}=0$ for some $s$, then $V_{j, A}^{1}=0$. In other words, each sector has a veto power. Accordingly an alternative with a high global value will likely be a strong one. 
The second formulation is based on the assumption that the sector set $S$ can be partitioned into $G$ groups, among which compensation is meaningful, while compensation is valid within each group:

\section{Formulation 2:}

$$
V_{j, A}^{2}=\prod_{g=1}^{G} \frac{1}{N_{g}} \sum_{s \in N_{g}} \frac{v_{j, A}^{s}}{v_{0}^{s}}
$$

where $\left|N_{g}\right|$ denotes the cardinality of the group $N_{g}$ of sectors.

The choice of the partition of $S$ is case-specific and should be done by decision-makers. It is also possible to consider many partitions, as will be shown later. Note that the veto power is still present, but it is now conferred on the sectors group.

In both the formulations the sector values are normalized to $v_{0}^{s}$ in order both to express the global value as improvement $(>1)$ or worsening $(<1)$ with respect to the reference alternative $A 0$ and to filter out the relevance of the absolute value of the sector values. In other words, the global value $V_{j, A}$ expresses the relative improvement with respect to $A 0$, rather than the absolute one.

\section{Comparing the classes}

Once the global values have been computed for all the alternatives of a given class, with either Formulation 1 or 2, they are presented in a plot (Fig. 2), where they appear on the y-axis and the alternatives on the $\mathrm{x}$-axis, the latter ranked for decreasing values. We will use the term ranking to refer to this plot. The comparison of the rankings of the different classes of alternatives is the support for decision-makers in choosing one class. The comparison may be based on qualitative metrics, which take into account:

- the dominance among the classes, i.e. the distances between the rankings, the existence and the position of their possible intersections;

- the dominance of each class with respect to the global value of alternative $A 0$;

- the ranking slopes.

We have not yet developed any formal procedure for comparing rankings, since its definition will require the analysis of a sufficient large number of cases. At the present time, we only have a few and therefore the comparison can only be based on intuition. For that reason we will consider it for the case of Lake Maggiore only.

\section{APPLICATION TO LAKE MAGGIORE WATER SYSTEM}

Formulation 1 can be directly applied to the planning problem, while Formulation 2 requires a previous definition of a partition of the sector set $S$. Two alternatives were considered:

Formulation 2a, partition by nations: it is the most natural one, since it reflects the point of view of the institutional decision-makers: Italy and Switzerland in our case. The rationale is that the governments are willing to accept trade-off among the sectors of their nation, but are reluctant to consider cross-border compensation;

Formulation 2b, partition by themes: energy, irrigation, economy, environment, upstream flood, downstream flood were the groups considered.

Figure 2 reports the ranking for classes $\mathbf{A}, \mathbf{B}$ and $\mathbf{C}$, using Formulation 1 (Fig. 2.1), Formulation 2a (Fig. 2.2a) and Formulation 2b (Fig. 2.2b) when the present (stationary) scenario is considered. The horizontal line is the global value of alternative $\mathrm{A} 0$.

On the basis of the qualitative metrics we mentioned in the previous section, it can be noted that: (1) In all the figures, class $\mathbf{C}$ outperforms the other two classes, while class $\mathbf{B}$ appears to be of no interest since the global values it produces are nearly always lower than the ones produced by class $\mathbf{A}$. This fact was confirmed also when the climate change scenarios were considered (see below), and therefore class $\mathbf{B}$ will not be shown in the following figures, to simplify the 


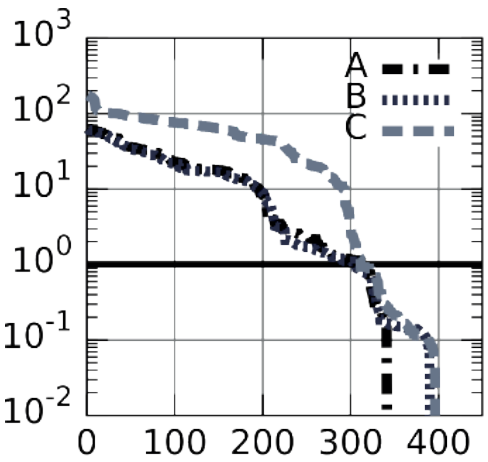

(1)

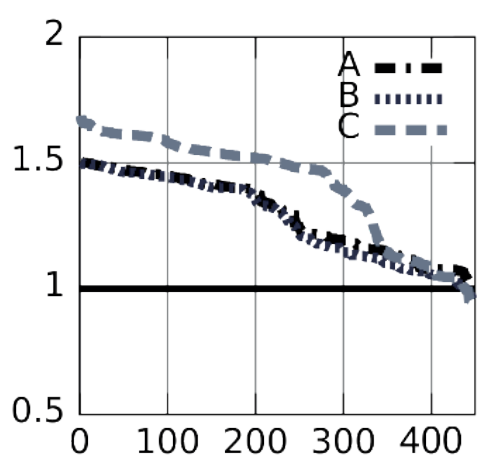

(2a)

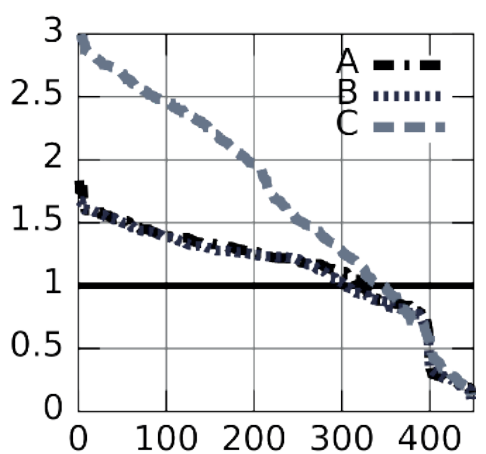

(2b)

Fig. 1 Stationary scenario: rankings with Formulation 1, 2a and 2b.

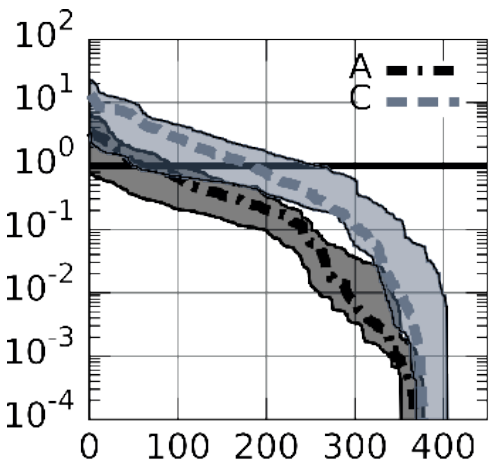

(1)

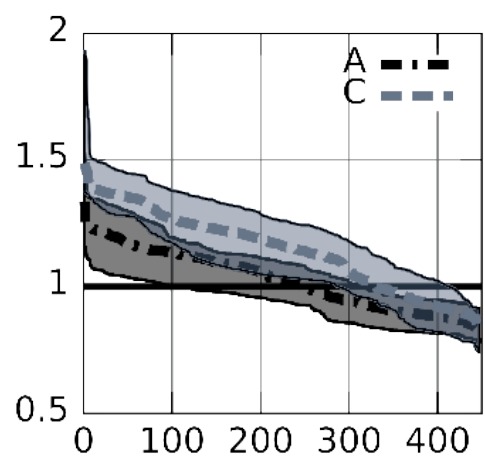

(2a)

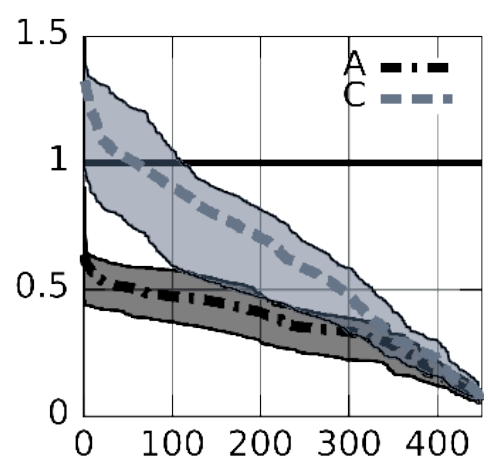

(2b)

Fig. 3 Climate change scenarios: rankings with Formulation 1, 2a and $2 \mathrm{~b}$.

visualization. (2) In all the classes at least 300 alternatives with a global value higher than $A 0$ exist and, when Formulation 2a is adopted, almost all the alternatives perform better. (3) In Fig. 2.1 some alternatives cause null satisfaction for at least one sector $\left(\mathrm{V}^{1}=0\right)$, i.e. the veto power is exerted, while the same does not occur for any group in Fig. 2.2a and 2.2b. (4) The steeper slopes of the rankings in Fig. 2.2b, with respect to Fig. 2.1 and 2.2a, reveals that the global value is more sensitive to the regulation policies when Formulation $2 \mathrm{~b}$ is adopted.

In conclusion, as far as the stationary scenario is considered, class $\mathbf{C}$ appears to be definitively superior to the other ones. That is due to the large discharge capacity it creates by dredging a stretch of the River Ticino and to the existence of management policies that prevent the downstream risks that such an increase might potentially create. This conclusion will now be tested for climate change situations.

Figure 3 shows the results for that case. For each class the central line is the median and the grey semi-transparent area the range within the 25th and the 75th percentile (uncertainty range) of the global values of the 10 alternatives that have the same ranking position in the 10 scenarios. On the basis of the qualitative metrics, one can note: (1) with all the formulations, class $\mathbf{C}$ outperforms class A: there are no intersections between the two medians and the uncertainty ranges are almost always separate, especially in the case of Fig. 3.1 and 3.2a. (2) A comparison of each of the three figures with the corresponding ones in the case of stationary scenario, reveals that the global values are now always lower than before (exception made for the extreme upper boundary of Fig. 3.2a). In other words the climate change will negatively affect sector satisfactions. Notwithstanding this fact, in class $\mathbf{C}$ there a significant number of alternatives that outperform alternative A0 still exists, while only few alternatives are such in class A when Formulation 1 is considered (Fig. 3.1) and none exists when considering Formulation 2b (Fig. 3.2b). (3) As for the stationary scenario, Formulation 2a (Fig. 3.2a) offers a more optimistic point of view: both for class $\mathbf{A}$ and class $\mathbf{C}$ a significant number 
of alternatives outperforms A0. (4) Finally, the rankings of the two classes in all the figures have quite steep slopes, highlighting once more that the choice of the regulation policy can make the difference.

In conclusion, class $\mathbf{C}$ appears to be definitively superior to the other ones.

\section{CONCLUSIONS}

We proposed a way for comparing classes of alternatives, characterized by the same structural interventions, under climate change conditions. The key element is the comparison of the rankings of the alternatives of the different classes with respect to the global values they produce, as computed by a suitably formulated global value function. Different formulations can be considered either to better represent the case-specific decision making structure or to perform a sensitivity analysis of the final result.

The application of this method in the Lake Maggiore case study indicates that the modification of the barrage and the dredging of the lake outlet (class $\mathbf{C}$ of alternatives) outperforms any modification of the regulation policy alone (class $\mathbf{A}$ ) or of both policy and regulation range (class C). Moreover class $\mathbf{C}$ is the only one that offers adaptation possibilities when climate change scenarios are considered. Using alternative global values formulations does not change this result, confirming its robustness.

Research is ongoing for strengthening the proposed method, namely for establishing a formal definition of the quantitative metrics for comparing rankings.

Acknowledgements The research was financed by the Action 2.1.4 in the context of Project STRADA-INTERREG Italy Switzerland Program, funded by the European Union.

\section{REFERENCES}

Bellman, R. (1957) Dynamic Programming. Princeton University Press.

Da Deppo, P. and Salandin, L. (2006). Studio degli effetti di una modifica dell'incile del Lago Maggiore e dell'opera di regolazione su portate e livelli del fiume Ticino e del fiume Po.Parte b: analisi del manufatto regolatore. Technical report, AIPO.

Intergovernmental Panel on Climate Change IPCC (2007) Climate Change 2007: The Scientific Basis. Cambridge University Press.

Keeney, R. L. and Raiffa, H. (1976) Decisions with Multiple Objectives: Preferences and Value Trade-offs. John Wiley \& Sons. Pal, Jeremy S., et al. (2007) Regional Climate Modeling for the Developing World: The ICTP RegCM3 and RegCNET. Bull. American Meteorological Society 88, 1395-1409.

Ravazzani, G, et al. (2008) Validation of FEST-WB, a continuous water balance distributed model for flood simulation. In: Proceedings of XXXI Italian Hydraulic and Hydraulic Construction Symposium.

Reed, M. S. (2008) Stakeholder participation for environmental management: A literature review. Biological Conservation 141(10), 2417-2431

Soncini-Sessa, R., Castelletti, A. and Weber, E. (2003) A DSS for planning and managing water reservoir systems. Environmental Modelling \& Software 18(5), 395-404.

Triantaphyllou, E. \& Baig, K. (2005) The impact of aggregating benefit and cost criteria in four MCDA methods. IEEE Transactions on Engineering Management 52(2), 213-226. 